\title{
A NEW HIGH-RESOLUTION PROCESSING METHOD FOR THE DECONVOLUTION OF OPTICAL COHERENCE TOMOGRAPHY SIGNALS
}

\author{
Thierry Blu, Herbert Bay and Michael Unser \\ Biomedical Imaging Group STI/IOA \\ Swiss Federal Institute of Technology Lausanne (EPFL) \\ CH-1015 Lausanne EPFL, Switzerland. \\ e-mail: $\left.\begin{array}{r}\text { thierry.blu } \\ \text { herbert.bay } \\ \text { michael.unser }\end{array}\right\}$ epfl.ch
}

\begin{abstract}
We show the feasibility and the potential of a new signal processing algorithm for the high-resolution deconvolution of OCT signals.

Our technique relies on the description of the measures in a parametric form, each set of four parameters describing the optical characteristics of a physical interface (e.g., complex refractive index, depth). Under the hypothesis of a Gaussian source light, we show that it is possible to recover the $4 K$ parameters corresponding to $K$ interfaces using as few as $4 K$ uniform samples of the OCT signal. With noisy data, we can expect the robustness of our method to increase with the oversampling rate-or with the redundancy of the measures.

The validation results show that the quality of the estimation of the parameters (in particular the depth of the interfaces) is narrowly linked to the noise level of the OCT measures - and not to the coherence length of the source light—and to their degree of redundancy.
\end{abstract}

\section{INTRODUCTION}

Optical Coherence Tomography (OCT) is a recent high-resolution imaging technique $[1,2,3]$. It is based on an interferometric measure in which a wave with low temporal coherence is split into two parts. One of the beams hits the object to image, is backscattered and guided to a photocaptor, while the second one acts as a reference and is guided to the photocaptor as well, where it is recombined with the object wave to yield interferences. Varying the pathlength of the reference wave, one is able to scan inside the object at depths such that the backscattered wave keeps some coherence with the incident one (see Fig. 1).

The OCT signal essentially carries the information of abrupt changes in the refractive index and the depth-resolution that can be attained is roughly half the coherence length of the reference wave [4]. In common experiments, this resolution is between $4 \mu \mathrm{m}$ and $16 \mu \mathrm{m} \mathrm{[5],} \mathrm{although} \mathrm{using} \mathrm{a} \mathrm{Fourier} \mathrm{deconvolution} \mathrm{technique}$ might improve this figure [6]

We propose here a direct signal processing method that is able to reach a resolution that does not depend on the coherence length, but rather on the noise level of the OCT measure. This highresolution parametric method is reminiscent of the retrieval of complex exponentials in a noisy signal, which is a standard signal pro-

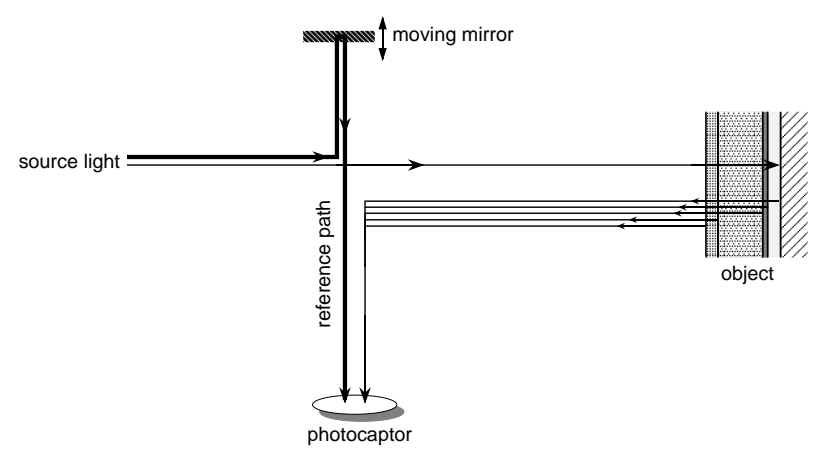

Fig. 1. Schematical overview of an OCT measure. The object is reduced to its interfaces as in the model used for this paper (see Section 2.2).

cessing problem, e.g., in mobile communications [7]. Our technique is actually exact under the assumption of a Gaussian coherence function; it is also "critical" in the absence of noise- the retrieval of $N$ parameters requires no more than $N$ samples of the interference pattern. The redundancy in the OCT measure, which may be quite high, can thus be exploited to reduce noise.

\section{MODELIZATION OF THE OCT SIGNAL}

\subsection{Standard Convolution Model}

An incident 1D complex wave $\psi(t-x / c)$, whose coherence function (and power spectrum density $S(\nu)=\hat{G}(\nu)$ ) is defined by

$$
G\left(t_{2}-t_{1}\right)=\left\langle\psi\left(t_{1}\right)^{*} \psi\left(t_{2}\right)\right\rangle
$$

illuminates an object that we want to image. We assume that this object is characterized by a linear time-invariant impulse response $h(t)$, which implies that it is the source of a backpropagating reflected wave $\psi_{\mathrm{R}}(t+x / c)$, where $\psi_{\mathrm{R}}=h * \psi$. This wave is guided to the photocaptor along a path of parameterized (variable) length $x_{1}$. The incident wave is also guided to this photocaptor along a path of (fixed) length $x_{0}$ and the average intensity resulting from 
the superposition of the two waves is measured

$$
\begin{aligned}
I\left(x_{1}-x_{0}\right) & =\left\langle\left|\psi\left(t+\frac{x_{0}}{c}\right)+\psi_{\mathrm{R}}\left(t+\frac{x_{1}}{c}\right)\right|^{2}\right\rangle \\
& =\int\left|1+\hat{h}(\nu) e^{2 i \pi \nu \frac{x_{1}-x_{0}}{c}}\right|^{2} S(\nu) \mathrm{d} \nu \\
& =\text { const }+2 \mathscr{R}\left\{h * G\left(\frac{x_{1}-x_{0}}{c}\right)\right\} .
\end{aligned}
$$

Finally, the signal that has to be processed is

$$
I_{0}(x)=2 \mathscr{R}\left\{h * G\left(\frac{x}{c}\right)\right\}
$$

We assume here that the power spectrum density of the incident light is Gaussian

$$
\begin{aligned}
S(\nu)= & \frac{1}{\sqrt{2 \pi} \Delta \nu} e^{-\frac{1}{2}\left(\frac{\nu-\nu_{0}}{\Delta \nu}\right)^{2}} \\
& \uparrow \mathscr{F} \\
G(t)= & e^{-\frac{(2 \pi \Delta \nu)^{2}}{2} t^{2}+2 i \pi \nu_{0} t}
\end{aligned}
$$

where $\nu_{0}$ is the central frequency of the source and $\Delta \nu$ is related to its temporal coherence length, $l_{c}$, through $l_{c}=\frac{\sqrt{2 \log 2}}{\pi} \frac{c}{\Delta \nu}$ [4].

\subsection{Multilayer Parametric Model}

As can be seen from (2) and (3), the OCT signal is a bandpass version of the signature $h(t)$ of the object. In particular, this means that attempting to reconstruct the low-pass portion of $h(t)$ from the OCT measurements, $I_{0}(t)$, is an ill-posed problem. This implies that it is only the transitional features of the object that are likely to be extracted, because they alone have a sufficiently large bandwidth to intersect the support of $S(\nu)$.

This is why we propose to model the object as a succession of interfaces between which the refractive index is constant. To simplify even more, we consider here the unidimensional case characterized by a complex refractive index

$$
n(x)=\left\{\begin{array}{l}
n_{0} \text { for } x<x_{1} \\
n_{k} \text { for } x_{k} \leq x<x_{k+1} \text { and } k=1 \ldots K-1 \\
n_{K} \text { for } x_{K} \leq x
\end{array}\right.
$$

and by the wave equation $y^{\prime \prime}(x)+\left(\frac{2 \pi \nu}{c}\right)^{2} n(x)^{2} y(x)=0$.

Then, the Fourier transform of the impulse response $h(t)$ is given by

$$
\hat{h}(\nu)=\sum_{k=1}^{K} a_{k} e^{-2 i \pi b_{k} \nu / c} \quad \text { for all }\left|\nu-\nu_{0}\right| \leq \frac{\Delta \nu}{2},
$$

neglecting multiple reflections. Here, a careful analysis of the wave equation provides the relation between $\left(a_{k}, b_{k}\right)$ and $\left(x_{k}, n_{k}\right)$.

As a consequence, the OCT signal can be parameterized as a sum of Gabor functions

$$
I_{0}(x)=2 \mathscr{R}\left\{\sum_{k=1}^{K} \alpha_{k} e^{-\frac{1}{2 \sigma^{2}}\left(x-\beta_{k}\right)^{2}}\right\}
$$

where $\alpha_{k}=a_{k} e^{-\frac{\nu_{0}^{2}}{2 \Delta \nu^{2}}}, \beta_{k}=b_{k}+i \frac{\nu_{0} c}{2 \pi \Delta \nu^{2}}$ and $\sigma=\frac{l_{c}}{2 \sqrt{2 \log 2}}$. The purpose of this paper is to present an exact method for identifying the parameters $\alpha_{k}$ and $\beta_{k}$ from a finite number of samples of $I_{0}(x)$.

\section{A HIGH-RESOLUTION METHOD}

We will from now on assume that we know the value of $l_{c}$, and hence, of $\sigma$. Moreover, we have access to the measurements $J_{l}=$ $I_{0}(l \Delta x)$ for $l=l_{0} \ldots l_{1}$ where $\Delta x$ is a sampling step. Thus, we have

$$
J_{l}=2 \mathscr{R}\left\{\sum_{k=1}^{K} \alpha_{k} e^{-\frac{\Delta x^{2}}{2 \sigma^{2}}\left(l-\frac{\beta_{k}}{\Delta x}\right)^{2}}\right\} .
$$

Multiplying left and right hand sides by $e^{\frac{\Delta x^{2}}{2 \sigma^{2}}(l-m)^{2}}$ yields

$$
\begin{aligned}
y_{l} & =e^{\frac{\Delta x^{2}}{2 \sigma^{2}}(l-m)^{2}} J_{l} \\
& =2 \mathscr{R}\left\{\sum_{k=1}^{K} \alpha_{k} e^{\frac{\Delta x^{2}}{2 \sigma^{2}}\left(\frac{\beta}{\Delta x}-m\right)\left(2 l-m-\frac{\beta}{\Delta x}\right)}\right\} \\
& =\sum_{k=1}^{K} \alpha_{k}^{\prime} z_{k}^{l}+\sum_{k=1}^{K} \alpha_{k}^{\prime *}\left(z_{k}^{*}\right)^{l}
\end{aligned}
$$

where we have defined $\alpha_{k}^{\prime}=\alpha_{k} e^{-\frac{\Delta x^{2}}{2 \sigma^{2}}\left(\frac{\beta}{\Delta x}-m\right)\left(m+\frac{\beta}{\Delta x}\right)}$ and $z_{k}=e^{\frac{\Delta x^{2}}{\sigma^{2}}\left(\frac{\beta}{\Delta x}-m\right)}$. The advantage of the last expression is that it takes a standard form that can be analyzed using a highresolution method [7].

\subsection{Finding the $z_{k}$ and $\alpha_{k}^{\prime}$}

Let $P(z)$ be the real polynomial that has the roots $z_{k}, z_{k}^{*}$

$$
P(z)=\prod_{k=1}^{K}\left(z-z_{k}\right)\left(z-z_{k}^{*}\right)=\sum_{k=0}^{2 K} p_{k} z^{k} .
$$

One can then easily verify that

$$
\forall l=l_{0}+2 K, \ldots, l_{1}, \quad \sum_{k=0}^{2 K} p_{k} y_{l-k}=0 .
$$

These linear equations are now written in a matrix form

$$
\underbrace{\left(\begin{array}{cccc}
y_{l_{0}+2 K} & y_{l_{0}+2 K-1} & \ldots & y_{l_{0}} \\
y_{l_{0}+2 K+1} & y_{l_{0}+2 K} & \ldots & y_{l_{0}+1} \\
y_{l_{0}+2 K+2} & y_{l_{0}+2 K+1} & \ldots & y_{l_{0}+2} \\
\vdots & \vdots & \vdots & \vdots \\
y_{l_{1}} & y_{l_{1}-1} & \ldots & y_{l_{1}-2 K}
\end{array}\right)}_{\text {A }} \underbrace{\left(\begin{array}{c}
p_{0} \\
p_{1} \\
p_{2} \\
\vdots \\
p_{2 K}
\end{array}\right)}_{\mathrm{P}}=0,
$$

where, $\mathbf{A}$ is a matrix having $\left(l_{1}-l_{0}-2 K+1\right)$ lines and $(2 K+1)$ columns. In particular, we see that, with as few as $4 K$ samples, we are able to reconstruct the polynomial $P(z)$. Extracting its roots provides the unknown values $z_{k}$.

Then, the parameters $\alpha_{k}^{\prime}$ are obtained by solving the linear Vandermonde system of equations obtained from (7)

$$
\left(\begin{array}{ccccc}
z_{1}^{l_{0}} & z_{1}^{* l_{0}} & \ldots & z_{K}^{l_{0}} & z_{K}^{* l_{0}} \\
z_{1}^{l_{0}+1} & z_{1}^{* l_{0}+1} & \ldots & z_{K}^{l_{0}+1} & z_{K}^{* l_{0}+1} \\
\vdots & \vdots & \vdots & \vdots & \vdots \\
z_{1}^{l_{1}} & z_{1}^{* l_{1}} & \ldots & z_{K}^{i_{1}} & z_{K}^{* l_{1}}
\end{array}\right)\left(\begin{array}{c}
\alpha_{1}^{\prime} \\
\alpha_{1}^{\prime *} \\
\vdots \\
\alpha_{K}^{\prime} \\
\alpha_{K}^{\prime *}
\end{array}\right)=\left(\begin{array}{c}
y_{l_{0}} \\
y_{l_{0}+1} \\
\vdots \\
y_{l_{1}}
\end{array}\right)
$$


In order to find the values $\alpha_{k}^{\prime}$, we need no fewer than $2 K$ measures $J_{l}$. On the whole, we conclude that we are able to reconstruct uniquely the $2 K$ complex-valued parameters $\left(\alpha_{k}^{\prime}, z_{k}\right)$ as soon as we are provided with $4 K$ real-valued uniform measures $J_{l}$. In the next subsection, we will see how it is possible to exploit the redundancy of these measures when they are corrupted by noise.

\subsection{The Noisy case}

Usually, the data are noisy and/or the model does not fit the data exactly. In this case, we propose first to enhance the signal, and then to solve the two linear systems of equations above in the leastsquare sense - in practice, this involves finding the smallest eigenvalue of the matrix $\mathbf{A}^{\mathrm{T}} \mathbf{A}$.

Our denoising technique uses the information that $y_{n}$ has the parametric form (7). In the absence of noise, the matrix

$$
\mathbf{B}_{N}=\left(\begin{array}{cccc}
y_{l_{0}+N} & y_{l_{0}+N-1} & \ldots & y_{l_{0}} \\
y_{l_{0}+N+1} & y_{l_{0}+N} & \ldots & y_{l_{0}+1} \\
y_{l_{0}+N+2} & y_{l_{0}+N+1} & \ldots & y_{l_{0}+2} \\
\vdots & \vdots & \vdots & \vdots \\
y_{l_{1}} & y_{l_{1}-1} & \ldots & y_{l_{1}-N}
\end{array}\right)
$$

is always of rank $2 K$ when $N \geq 2 K$. Its decomposition in singular values $\mathbf{B}_{N}=\mathbf{Q} \boldsymbol{\Delta} \mathbf{R}^{\mathrm{T}}$ therefore involves a diagonal matrix $\Delta$ with only $2 K$ nonzero values. Our direct denoising method can now be described as follows:

- First, choose a denoising length $N$. The longer it is, the larger the reduction of the noise-but also, the more computationally expensive it is.

- Second, perform the decomposition of the noisy measurement matrix $\mathbf{B}_{N}$ in singular values.

- Third, build the "denoised" diagonal matrix $\hat{\Delta}$ by keeping only the $2 K$ largest coefficients of $\boldsymbol{\Delta}$; then reconstruct the denoised measurement matrix $\hat{\mathbf{B}}_{N}=\mathbf{Q} \hat{\Delta} \mathbf{R}^{\mathrm{T}}$.

- Last, since every column of $\mathbf{B}_{N}$ provides an estimate of the signal, build a denoised version of the signal by averaging over the estimates of $y_{l}$ obtained from the $N+1$ columns of $\hat{\mathbf{B}}_{N}$.

This denoising technique has proved extremely efficient on the test signals that we have analyzed. For instance, with 350 samples, two Gaussians to retrieve, and a denoising length of $N=50$, the signal to noise ratio increase usually exceeds $16 \mathrm{~dB}$.

\section{VALIDATION RESULTS}

We have synthetized 353 uniform samples of an OCT signal simulating the reflection of two interfaces separated by a distance of $2.66 \mu \mathrm{m}$ and inducing a wavelength shift from $1300 \mathrm{~nm}$ to $1302 \mathrm{~nm}$ and $1339 \mathrm{~nm}$, respectively. The coherence length for the Gaussian source is $20 \mu \mathrm{m}$, so the equivalent depth resolution is $10 \mu \mathrm{m}$. We have added a Gaussian white noise ranging from $6 \mathrm{~dB}$ to $60 \mathrm{~dB}$ and have applied our algorithm to these 353 samples. An instance of this noisy simulated OCT signal is shown in Fig. 2.

The main retrieved parameters (position and frequency) are shown in the scatterplots of Figs. 3 and 4. Using these estimated parameters we reconstructed a signal that is much closer to the original noise-free signal than to the noisy one. More precisely, our reconstruction increases the signal to noise ratio by $16 \mathrm{~dB}$ on average as shown in Fig. 5. This gain is identical to $\sqrt{\text { redundancy }}$ which is what we would expect from an optimal estimator.
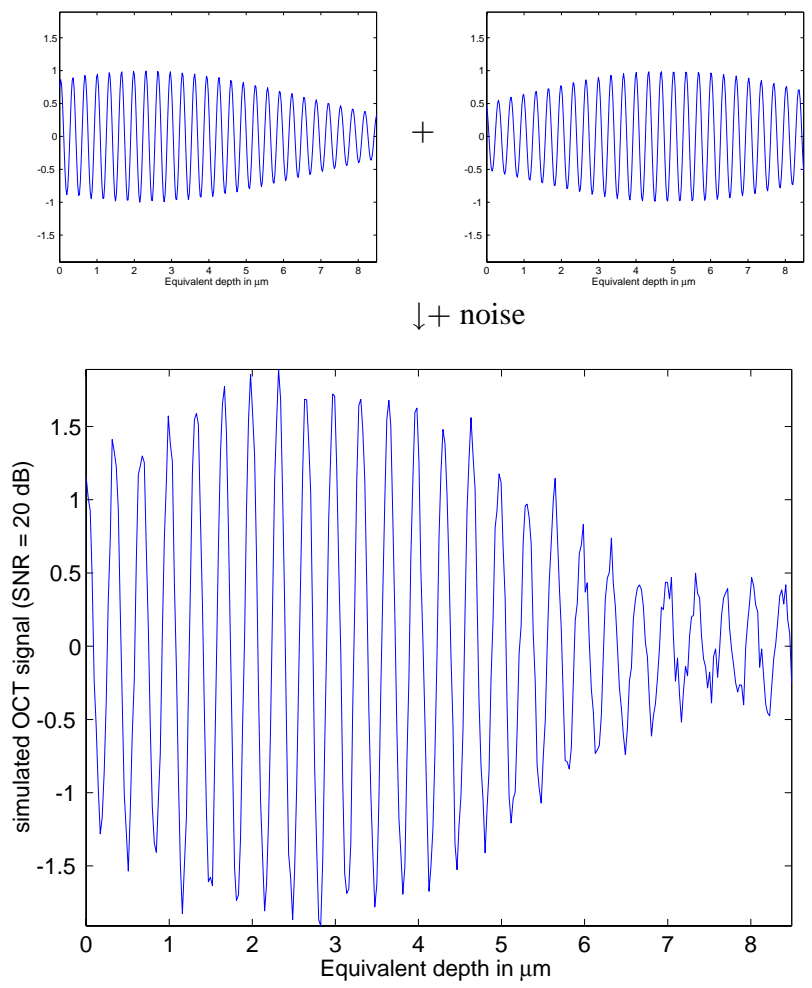

Fig. 2. Simulated OCT signal-Gaussian light source at $1300 \mathrm{~nm}$ with $20 \mu \mathrm{m}$ temporal coherence length — composed of two modulated Gaussians and corrupted with $20 \mathrm{~dB}$ noise. Here, the "standard" axial resolution is $20 \mu \mathrm{m} / 2=10 \mu \mathrm{m}$.

\section{CONCLUSION}

We have presented a new high-resolution signal processing method for the deconvolution of OCT signals. In particular, we have shown that the resolution of our method is only limited by the amount of noise that is present in the measures: less noisy data yield higher resolved structures. Moreover, our technique is versatile as it requires only a finite number of samples for the exact retrieval of the parameters that describe the OCT signal. It may be used either when few but noise-free data are available, or in the case of oversampled noisy measurements.

\section{Acknowledgements}

We want to thank Profs R.P. Salathé and T. Lasser for the fruitful discussions that led to this algorithm. We also thank M. Ducros, M. Laubscher and L. Frœhly for providing us with OCT data and guiding us through their OCT experiments.

Finally, we would like to mention that it is through a collaboration with Prof. M. Vetterli and P. Marziliano on a completely different problem, but requiring a similar solution, that the idea of this high-resolution method arose [8].

\section{REFERENCES}

[1] D. Huang, E.A. Swanson, C.P. Lin, J.S. Schuman, W.G. Stinson, W. Chang, M.R. Hee, T. Flotte, K. Gregory, C.A. Pu- 
liafito, and J.G. Fujimoto, "Optical coherence tomography," Science, vol. 254, pp. 1178-1181, 1991.

[2] A.F. Ferchner, "Optical coherence tomography," J. Biomed. Optics, vol. 1, no. 2, pp. 157-173, April 1996.

[3] J.M. Schmitt, "Optical coherence tomography (OCT): A review," IEEE Trans. Sel. Topics in Quantum Electron., vol. 5, no. 4, pp. 1205-1215, July/August 1999.

[4] A.F. Ferchner and C.K. Hitzenberger, "Optical coherence tomography in medicine," in International Trends in Optics and Photonics ICO IV, T. Asakura, Ed. Springer, 1999.

[5] M.E. Brezinski and J.G. Fujimoto, "Optical coherence tomography: High-resolution imaging in nontransparent tissue," IEEE Trans. Sel. Topics in Quantum Electron., vol. 5, no. 4, pp. 1185-1192, July/August 1999.

[6] R.K. Wang, "Resolution improved optical coherence-gated tomography for imaging through biological tissues," J. Modern Optics, vol. 46, no. 13, pp. 1905-1912, 1999.

[7] P. Stoica and R. Moses, Introduction to Spectral Analysis, Prentice Hall, 2000.

[8] M. Vetterli, P. Marziliano, and T. Blu, "Sampling signals with finite rate of innovation," IEEE Trans. Signal Process., 2001, to appear.

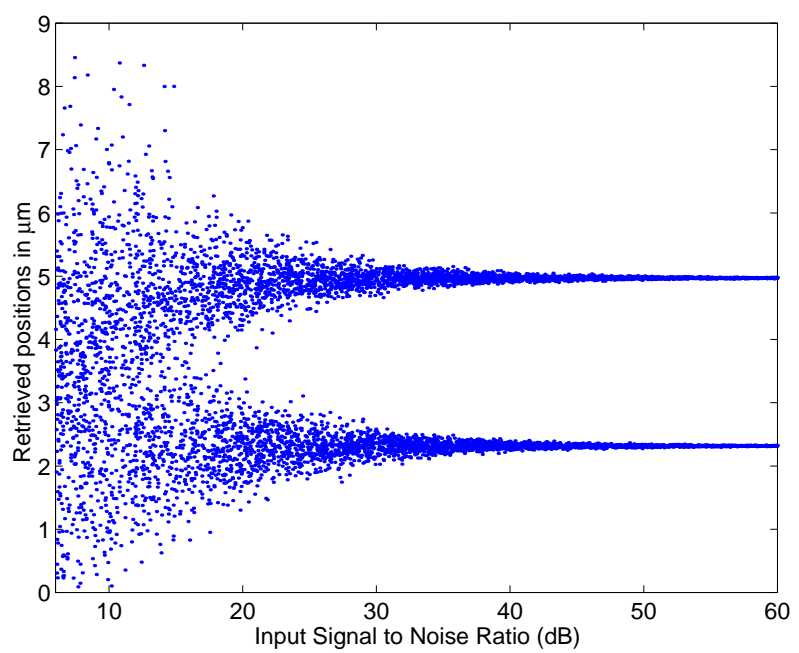

Fig. 3. Positions of the interfaces as a function of the input noise. Notice that the depth resolution depends only on the noise level, not on the coherence length which is here a constant.



Fig. 4. Retrieved wavelengths as a function of the input noise. Observe that the frequency resolution does not depend on the coherence length—nor, actually, on the length of the signal.

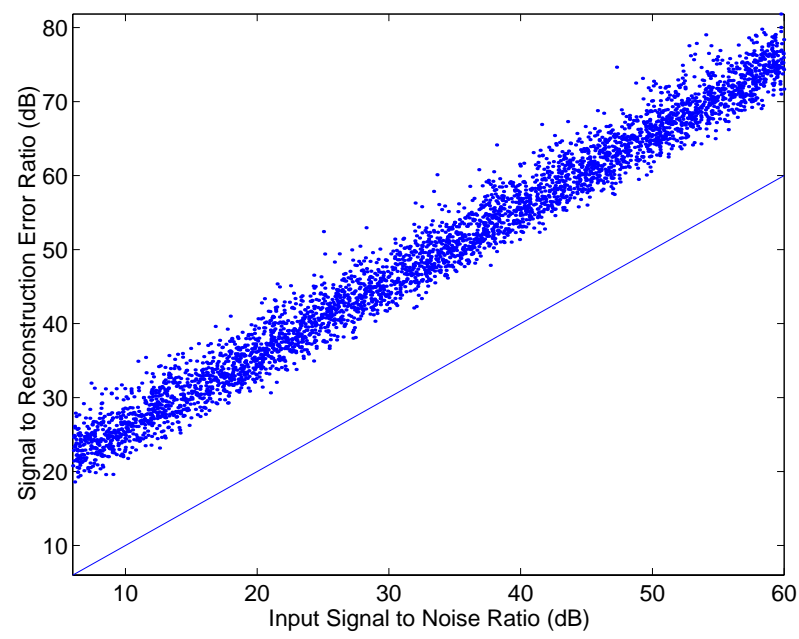

Fig. 5. Error between the original signal and the reconstruction using the retrieved noisy parameters (in ordinate) as a function of the level of input noise (in abscissa). To ease the comparison, we have drawn the $y=x$ line. Note that the level of output noise is reduced dramatically ( $16 \mathrm{~dB}$ on average) which shows the efficiency of our method in exploiting the redundancy of the measures. 